\title{
Analytical Performance Evaluation of Distributed Multicast Algorithms for Directional Communications in WANETs
}

\author{
Song Guo ${ }^{1}$, Oliver Yang ${ }^{2}$, and Victor Leung ${ }^{3}$ \\ ${ }^{1}$ Computer Science, University of Northern British Columbia, Canada \\ sguo@unbc.ca \\ ${ }^{2}$ School of Information Technology and Engineering, University of Ottawa, Canada \\ yang@site.uottawa.ca \\ ${ }^{3}$ Electrical and Computer Engineering, University of British Columbia, Canada \\ vleung@ece.ubc.ca
}

\begin{abstract}
Two distributed algorithms DMMT-OA and DMMT-DA have been recently proposed to maximize the multicast lifetime for directional communications in wireless ad-hoc networks. The experimental results have shown their superior performance than other centralized algorithms; however, their theoretical performance in terms of approximation ratio is still unknown. In this paper, we use graph theoretic approach to derive the approximation ratio for both algorithms. Furthermore, we have discovered by the first time that both ratios are bounded by a constant number.
\end{abstract}

Keywords: Wireless Ad Hoc Networks, Approximation Algorithm, Multicast, Distributed Algorithm, Directional Communications.

\section{Introduction}

Over the last few years, energy efficient communication in Wireless Ad Hoc Networks (WANETs) with directional antennas has received more and more attention. This is because directional communications can save transmission power by concentrating RF energy where it is needed [1. On the other hand, the broadcast / multicast communication is also an important issue as many routing protocols for WANETs need this mechanism to maintain the routes between nodes. Therefore, one would be interested in finding an algorithm that would provide the maximum lifetime to the multicast session. The optimization metric is typically defined as the duration of the network operation time until the battery depletion of the first node in the network [2] [3.

Some work has considered maximizing the network lifetime in a WANET with omni-directional antennas for a broadcast session, e.g., [2] 3] [4], or a multicast session, e.g., [5] 6] [7]. The same problem with directional antennas has been studied in 1 9 10 11] and shown to be a NP-hard problem 11. The only exact solution for such difficult problem is the MILP formulation presented in [10]. 
We note that all the solutions in [1][10]11] are centralized, meaning that at least one node needs global network information in order to construct an energy efficient multicast tree. Sometimes, this centralized approach is impractical for resource-constrained WANETs.

The most desirable work has been presented in [9], in which two distributed maximum-lifetime algorithms have been proposed for directional communications. Simulation results have also shown that they outperform other centralized multicast algorithms, e.g., 1], significantly. However, their theoretical performance in terms of approximation ratio is still unknown so far. In this paper, we would like to explore the approximation ratio for these two heuristic algorithms from a graph theoretic approach.

\section{Network Model}

We model our wireless ad hoc network as a simple directed graph $G$ with a finite node set $N$ and an arc set $A$ corresponding to the unidirectional wireless communication links. We assume a free-space propagation model and an adaptive antenna model, in which the antenna at each node $v$ can switch its orientation to any desired direction with transmission power uniformly distributed across its adjustable beamwidth $\theta_{v}$ between constant numbers $\theta_{\min }$ and $2 \pi$. The transmission power $p_{v u}$ to support a link $(v, u)$ separated by a distance $r_{v u}\left(r_{v u}>1\right)$ is therefore

$$
p_{v u}=\frac{\theta_{v}}{2 \pi} \cdot r_{v u}^{\alpha}
$$

We consider a source-initiated multicast with multicast members $M=\{s\} \cup D$, where $s$ is the source node and $D$ is the set of destination nodes. All the nodes involved in the multicast form a multicast tree rooted at the node $s$, i.e., a rooted tree $T_{s}$, with a tree node set $N\left(T_{s}\right)$ and a tree arc set $A\left(T_{s}\right)$. Let the battery supply $\varepsilon_{v}$ be the energy level associated with each node $v$. We assign the tree arc weight function $w_{v u}$ as the reciprocal of the maximum lifetime of the arc $(v, u)$ as defined as follows.

$$
w_{v u}\left(\theta_{v}\right)=\frac{\theta_{v} \cdot r_{v u}^{\alpha}}{2 \pi \cdot \varepsilon_{v}}
$$

Note that the beamwidth $\theta_{v}$ applied by node $v$ in the multicast tree $T_{s}$ is a function of node $v$ 's child node set $c_{v}$, i.e., $\theta_{v}=\varphi\left(c_{v}\right)$ and $c_{v}=\{u \mid(v, u) \in$ $\left.A\left(T_{s}\right)\right\}$. Such function $\varphi\left(c_{v}\right)$ is defined as the smallest possible beamwidth at node $v$ in the range between $\theta_{\min }$ and $2 \pi$ to provide beam-coverage of $c_{v}$ in the tree $T_{s}$.

Let $\Omega_{M}$ be the family of all rooted multicast trees spanning nodes in $M$. It has been shown in 9 that the maximum-lifetime multicast problem is equivalent to the min-max tree problem, which is to determine a directed tree $T_{s}$ spanning all the multicast members (i.e., $M \subseteq N\left(T_{s}\right)$ such that the maximum arc weight is minimized, i.e.,

$$
\min _{T_{s} \in \Omega_{M}} \max _{(v, u) \in A\left(T_{s}\right)} w_{v u}\left(\theta_{v}\right)
$$




\section{Two Approximation Algorithms}

Two distributed maximum-lifetime algorithms DMMT-OA (Distributed MinMax Tree algorithm for Omnidirectional Antennas) and DMMT-DA (Distributed Min-Max Tree algorithm for Directional Antennas) 9] have been proposed for directional communications. It has been proved that the degenerate versions of both distributed algorithms for omni-directional antennas are globally optimal. For both algorithms, the multicast tree is constructed in a distributed and incremental manner. Initially, the multicast tree $T_{s}$ only contains the source node. It then iteratively performs the following Search-and-Grow procedure until the tree contains all the nodes in $M$.

Search-and-Grow: Find the link $(v, u)$ connecting tree node set and non-tree node set with minimum weight $w_{v u}$, and then include it into the multicast tree. Consequently, the tree $T_{s}$ would grow by including as many non-tree links $(x, y)$ as possible into the multicast tree if $w_{x y} \leq w_{v u}$ until no more such links can be found.

The DMMT-OA algorithm disregards the beamwidth in the tree construction process, assuming using omnidirectional antennas, i.e., $w_{v u}\left(\theta_{v}\right)=w_{v u}(2 \pi)$ for each $\operatorname{arc}(v, u)$ in the graph. After the tree $T_{s}$ is constructed, each internal node $v$ set its antennas beamwidth to $\varphi\left(c_{v}\right)$. Unlike the arc weights $w_{v u}(2 \pi)$ in the DMMT-OA, which remain unchanged throughout the execution of the algorithm, the D-DPMT algorithm dynamically updates the weights $w_{v u}\left(\theta_{v}\right)$ at each step to reflect the changes of the smallest beamwidth $\theta_{v}$. In the following theorems, we shall show that both algorithms have bounded approximation ratios $\rho_{1}$ and $\rho_{2}$, respectively. The technical detail of the proofs can be found in [12].

Theorem 1. The DMMT-OA algorithm has a bounded approximation ratio $\rho_{1}$,

$$
\rho_{1} \leq \frac{\varphi_{1}}{\theta_{\min }},
$$

where $\varphi_{1}$ is the minimum beamwidth applied by the transmitting node $v$ of the $\operatorname{arc}(v, u)$ in the final multicast tree $T_{s}$ obtained by DMMT-OA, i.e., $\varphi_{1} \equiv \varphi\left(c_{v}\right)$, in which $(v, u)$ satisfies

$$
w_{v u}\left(\varphi\left(c_{v}\right)\right)=\max _{(x, y) \in A\left(T_{s}\right)} w_{x y}\left(\varphi\left(c_{x}\right)\right) .
$$

Let $T_{s}$ be the final multicast tree obtained from the DMMT-DA algorithm. We use $T_{s}^{k}$ and $c_{v}^{k}$ to denote the partially constructed tree rooted at $s$ and the childnode set of node $v$, respectively, after the $k$-th node is added into the tree, where $k=0,1, \ldots, n-1$. We assume that the ending node $u$ of the bottleneck arc $(v, u)$ is the $i$-th node added into the tree $T_{s}$ and the node chosen at the beginning of the same search-and-grow cycle as arc $(v, u)$ is the $j$-th $(j \leq i)$ one that was added into the tree. Note that $c_{v}$ excludes all pruned nodes from $c_{v}^{n-1}$.

Theorem 2. The DMMT-DA algorithm has a bounded approximation ratio $\rho_{2}$,

$$
\rho_{2} \leq \frac{\varphi_{2} \cdot \varphi\left(c_{v}\right)}{\theta_{\min } \cdot \varphi\left(c_{v}^{i}\right)},
$$


where $\varphi_{2}$ is the smallest beamwidth applied by the transmitting node $v^{\prime}$ before the $j$-th node added into the tree such that there exists an arc $\left(v^{\prime}, u^{\prime}\right)$ with minimum weight $w_{v^{\prime} u^{\prime}}(2 \pi)$ connecting node sets $X$ and $N-X$, where $X=N\left(T_{s}^{j-1}\right)$, i.e., $\varphi_{2} \equiv \varphi\left(c_{v^{\prime}}^{j-1} \cup\left\{u^{\prime}\right\}\right)$, in which $\left(v^{\prime}, u^{\prime}\right)$ satisfies

$$
w_{v^{\prime} u^{\prime}}(2 \pi)=\min _{x \in X, y \in N-X} w_{x y}(2 \pi) .
$$

\section{Conclusion}

The main contribution of this paper is to provide the fact that both DMMT-OA and DMMT-DA algorithms have bounded approximation ratios. These results would help us understand why they have superior performance than other proposals in simulation experiments.

\section{References}

1. J. E. Wieselthier, G. D. Nguyen, et al: Energy-Limited Wireless etworking with Directional Antennas: The Case of Session-Based Multicasting. IEEE INFOCOM, New York, 2002, pp. 190 - 199.

2. I. Kang and R. Poovendran: Maximizing Static Network Lifetime of Wireless Broadcast Adhoc Networks. IEEE ICC, Alaska, 2003, pp. 2256 - 2261.

3. A. K. Das, R. J. Marks II, et al: MDLT: a polynomial time optimal algorithm for maximization of time-to-first-failure in energy-constrained broadcast wireless networks. IEEE Globecom, San Francisco, 2003, pp. 362 - 366.

4. I. Kang and R. Poovendran: On the Lifetime Extension of Energy-Efficient Multihop Broadcast Networks. World Congress on Computational Intelligence, Honolulu, 2002.

5. M. X. Cheng, J. Sun, and et al: Energy-efficient Broadcast and Multicast Routing in Ad Hoc Wireless Networks. IEEE IPCCC, Phoenix, 2003, pp.87 - 94.

6. B. Floren, P. Kaski, and et al: Multicast time maximization in energy constrained wireless networks. Workshop on Foundations of Mobile Computing, San Diego, 2003, pp. 50 - 58.

7. L. Georgiadis: Bottleneck multicast trees in linear time. IEEE Communications Letters, 7(11), Nov. 2003, pp. 564 - 566.

8. S. Guo, V. Leung and O. Yang: A Scalable Distributed Multicast Algorithm for Lifetime Maximization in Large-scale Resource-limited Multihop Wireless Networks. ACM IWCMC, Vancouver, 2006, pp. 419 - 424.

9. S. Guo, V. Leung and O. Yang: Distributed Multicast Algorithms for Lifetime Maximization in Wireless Ad Hoc Networks with Omni-directional and Directional Antennas. IEEE Globecom, San Francisco, 2006.

10. S. Guo and O. Yang: Optimal Tree Construction for Maximum Lifetime Multicasting in Wireless Ad-hoc Networks with Adaptive Antennas. IEEE ICC, Seoul, 2005, pp. $3370-3374$.

11. Y. Hou, Y. Shi, H. D. Sherali, and J. E. Wieselthier: Online lifetime-centric multicast routing for ad hoc networks with directional antennas. IEEE INFOCOM, Miami, 2005, pp. $761-772$.

12. S. Guo: Proofs of the Approximation Ratio Bounded Algorithms for the Maximum Lifetime Multicast Problems in WANETs. Technical Reports, http://web.unbc. $\mathrm{ca} /{ }^{\sim}$ sguo/publications. 